\title{
Integrin Activation Promotes Axon Growth on Inhibitory Chondroitin Sulfate Proteoglycans by Enhancing Integrin Signaling
}

\author{
Chin Lik Tan, ${ }^{1}$ Jessica C. F. Kwok, ${ }^{1}$ Rickie Patani, ${ }^{1,2}$ Charles ffrench-Constant, ${ }^{3}$ Siddharthan Chandran, ${ }^{1,2,4}$ \\ and James W. Fawcett ${ }^{1}$ \\ ${ }^{1}$ Centre for Brain Repair, Department of Clinical Neurosciences, University of Cambridge, Cambridge CB2 0PY, United Kingdom, ${ }^{2}$ The Anne McLaren \\ Laboratory for Stem Cell Biology and Regenerative Medicine, Cambridge CB2 0SZ, United Kingdom, ${ }^{3}$ Centre for Multiple Sclerosis Research, MRC Centre \\ for Regenerative Medicine, University of Edinburgh, Queens' Medical Research Institute, Edinburgh EH16 4TJ, United Kingdom, and ${ }^{4}$ Centre for Clinical \\ Brain Sciences, MRC Centre for Regenerative Medicine, University of Edinburgh, Edinburgh EH16 4SB, United Kingdom
}

Chondroitin sulfate proteoglycans (CSPGs) are upregulated after CNS lesions, where they inhibit axon regeneration. In order for axon growth and regeneration to occur, surface integrin receptors must interact with surrounding extracellular matrix molecules. We have explored the hypothesis that CSPGs inhibit regeneration by inactivating integrins and that forcing integrins into an active state might overcome this inhibition. Using cultured rat sensory neurons, we show that the CSPG aggrecan inhibits laminin-mediated axon growth by impairing integrin signaling via decreasing phosphorylated FAK (pFAK) and pSrc levels, without affecting surface integrin levels. Forcing integrin activation and signaling by manganese or an activating antibody TS2/16 reversed the inhibitory effect of aggrecan on mixed aggrecan/ laminin surfaces, and enhanced axon growth from cultured rat sensory neurons (manganese) and human embryonic stem cell-derived motoneurons (TS2/16). The inhibitory effect of Nogo-A can also be reversed by integrin activation. These results suggest that inhibition by CSPGs can act via inactivation of integrins, and that activation of integrins is a potential method for improving axon regeneration after injury.

\section{Introduction}

Chondroitin sulfate proteoglycans (CSPGs) are upregulated at sites of damage in the CNS, inhibiting axon regeneration (Tang et al., 2003; Silver and Miller, 2004). Of the several inhibitory CSPGs that are present, aggrecan has been shown to be strongly inhibitory to neurite outgrowth from several types of cultured neurons (Borisoff et al., 2003; Chan et al., 2008). It may act via several mechanisms, including the transmembrane protein tyrosine phosphatase $\sigma(\mathrm{PTP} \sigma)$ receptor (Shen et al., 2009) or the epidermal growth factor receptor (EGFR) (Koprivica et al., 2005), and through a variety of downstream signaling cascades, such as Rho/ROCK pathway or protein kinase C (PKC) (Monnier et al., 2003; Sivasankaran et al., 2004). Aggrecan is synthesized primarily by neurons and is endogenously present in the CNS (Asher et al., 1995; Li et al., 1996). It is also modulated after a CNS injury at the lesion site, where it is secreted by reactive astrocytes (Silver and Miller, 2004; Afshari et al., 2010).

Received Dec. 14, 2010; revised Feb. 17, 2011; accepted March 2, 2011.

Author contributions: C.L.T., J.C.F.K., R.P., C.f-C., S.C., and J.W.F. designed research; C.L.T., J.C.F.K., and R.P. performed research; C.L.T. and J.C.F.K. analyzed data; C.L.T. wrote the paper.

This work was funded by grants from the Medical Research Council, the Henry Smith Charity, the John and Lucille van Geest Foundation, the European Union Framework 6 Network of Excellence NeuroNE, the European Union Framework 7 Programmes Spinal Cord Repair, and the MND Association. C.L.T. was supported by the Merck, Sharpe, and Dohme Fund; and R.P. by the Sir David Walker Fellowship, the Beverly and Raymond Sackler Fellowship, and the Wellcome Trust Clinical Research Training Fellowship.

The authors declare no competing financial interests.

Correspondence should be addressed to James W. Fawcett, Centre for Brain Repair, Department of Clinical Neurosciences, University of Cambridge, Cambridge CB2 OPY, UK. E-mail: jf108@cam.ac.uk.

DOI:10.1523/JNEUROSCI.0008-11.2011

Copyright $\odot 2011$ the authors $\quad 0270-6474 / 11 / 316289-07 \$ 15.00 / 0$
Growth cone migration and axon extension require an adhesion molecule as a physical link between the cell and its substrate (Long and Lemmon, 2000; Previtali et al., 2001; Song and Poo, 2001). For interaction with molecules present in the extracellular matrix (ECM), the main receptors are integrins, which function as both adhesion and signaling molecules (Hynes, 2002; Lemons and Condic, 2008). Integrins are $\alpha \beta$ heterodimeric transmembrane receptors, whose functions are regulated by two different but related processes, "inside-out" and "outside-in" signaling. The former (also termed "integrin activation") indicates a change from a low ligand-binding affinity (inactive) conformation to a high-affinity (active) one. "Outside-in" signaling refers to the signaling cascades propagated intracellularly upon ligand-integrin binding. In the nervous system, integrins are involved in axon guidance, neurite extension, synaptic plasticity, and axon regeneration [for review, see Reichardt et al. (1989) and Lemons and Condic (2008)]. Previous work has indicated that the CNS inhibitory molecule Nogo-A exerts some of its effects through integrin inactivation (Hu and Strittmatter, 2008). Here we have investigated whether the effect of aggrecan in impairing axon regeneration could also be mediated though an integrin-related mechanism, and whether manipulation of integrins can allow axons to overcome this inhibition.

\section{Materials and Methods}

Dorsal root ganglion neuron culture. Dorsal root ganglia (DRGs) were dissected from adult male Sprague Dawley rats ( $\sim 3$ months). The neurons were dissociated with collagenase and $0.1 \%$ trypsin, centrifuged through a $15 \%$ bovine serum albumin (BSA) density gradient, and cul- 
tured in DRG culture medium [DMEM, insulin-transferrin-selenium $(1 \times)$, penicillinstreptomycin-fungizone $(1 \times)$, and NGF $(10$ $\mathrm{ng} / \mathrm{ml})]$. Cultures were kept for $20 \mathrm{~h}$ at $37^{\circ} \mathrm{C}$ in $7 \% \mathrm{CO}_{2}$. Where relevant, $500 \mu \mathrm{M}$ manganese was added only after neurons were attached to coverslips ( $\sim 2 \mathrm{~h}$ after plating). For removing the glycosaminoglycan (GAG) chains on aggrecan, chondroitinase ABC (Sigma, C2905, $0.1 \mathrm{U} / \mathrm{ml}$ ) was added to aggrecan for $1 \mathrm{~h}$ at $37^{\circ} \mathrm{C}$ before using it for coating/treatment on DRG cultures.

Human embryonic stem cell-differentiated motoneuron culture. Human embryonic stem cell (hESC) lines (H9 from the WiCell Research Institute and HuES9 from hES facility, Harvard University) between passages 50 and 90 were used for this study. hESC culture and neural induction were performed using a protocol adapted from Patani et al. (2009). Briefly, hESCs were propagated in defined medium supplemented with $8 \mathrm{ng} / \mathrm{ml} \mathrm{FGF2,} 10 \mathrm{ng} / \mathrm{ml}$ Activin (Harrington et al., 2006), and $10 \mathrm{ng} / \mathrm{ml}$ insulin. To generate neural precursor cells (NPCs), hESCs were enzymatically and mechanically dissociated before being plated in chemically defined medium, comprising $50 \%$ Iscove's modified Dulbecco's medium (IMDM), 50\% F12 and GlutaMAX, supplemented with $1.75 \mathrm{~mm}$ human recombinant insulin, $0.38 \mathrm{~mm}$ transferrin, $450 \mu \mathrm{M}$ monothioglycerol, $10 \mu \mathrm{l} / \mathrm{ml}$ lipids, and $5 \mathrm{mg} / \mathrm{ml}$ BSA fraction $\mathrm{V}$ on an orbital shaker. For spinal motoneuron specification, hESC-NPCs were cultured in 10-20 ng/ml FGF2 and $0.5 \mu \mathrm{M} R A / 1 \mu \mathrm{M}$ purmorphamine. For terminal differentiation, hESC-NPCs were cultured on laminin-coated coverslips in DMEM supplemented with B27, PSF, $10 \mathrm{ng} / \mathrm{ml} \mathrm{BDNF}$, and 10 ng/ml GDNF.

For experiments involving the TS2/16 antibody (ATCC-HB243, Hybridoma Bank), hESCNPCs were plated on coverslips coated with laminin or aggrecan-laminin and cultured in the differentiation medium (as described above) for $2 \mathrm{~d}$ at $37^{\circ} \mathrm{C}$. Then, TS2/16 was added at 1:3 dilution, and the cultures were kept for another day.

Postfixation immunostaining. Cell cultures on coverslips were fixed with $4 \%$ paraformaldehyde (PFA), permeabilized with $0.1 \%$ Triton $\mathrm{X}-100$, blocked with goat serum, and incubated with primary antibodies at $4^{\circ} \mathrm{C}$ overnight. Then, coverslips were incubated with secondary antibodies for $1 \mathrm{~h}$ before mounting on slides. Primary antibodies were used against pY397 FAK (Biosource, 44-624G, 1:100), pY861 FAK (Abcam, ab4804, 1:100), pY418 Src (Invitrogen, 44-660G, 1:100), FAK (Biosource, AHO0502, 1:100), $\beta$ III tubulin (Sigma, T8660, 1:400), neurofilament-H (Biomol, NA1211, 1:1000), laminin (Sigma, L9393, 1:100), and CS-56 (Sigma, C8035, 1:500).

Live immunostaining. 9EG7 antibody (BD Pharmingen, 550531, 1:50 in culture medium) was added to the cultures for $15 \mathrm{~min}$ at $37^{\circ} \mathrm{C}$. After washing once with culture medium, the cultures were fixed with $4 \%$ PFA and incubated with FITC-conjugated goat anti-rat antibody for $1 \mathrm{~h}$ before mounting on slides.

Western blotting. Cell lysates from manganese- or aggrecan-treated DRG cultures were lysed and collected in RIPA buffer (Roche), supplemented with protease inhibitor and phosphatase inhibitor cocktails (Roche). The protein concentrations were then determined using BCA Protein Assay Kit (Pierce). Protein extracts were subjected to SDS-PAGE and blotted onto polyvinylidene difluoride (Hybond-P) membranes (GE Healthcare). Membranes were blocked with 5\% skimmed milk in TBS-T, before incubation with rabbit anti-pY397 FAK (Biosource) or mouse anti-FAK (Millipore) antibodies at $4^{\circ} \mathrm{C}$ overnight. This was followed by incubation in HRP-conjugated anti-rabbit or anti-mouse antibodies at room temperature for $1 \mathrm{~h}$. Protein bands were visualized using ECLdetecting reagents (GE Healthcare).

Axon growth assay. For adult DRG neurons, two parameters were quantified: (1) percentage of neurons with axons longer than the cell body diameter; (2) average of the longest axons extended by each neuron. For hESC-derived motoneurons, the lengths of $\beta$ III tubulin-positive (for differentiated neurons) axons extended by motoneurons were measured.

Quantitative immunofluorescence. At least 20 axons per coverslip were first selected at random and imaged. An area of axon $(>30 \mu \mathrm{m}$ long $)$ was then traced, and the fluorescence intensity of immunostaining was analyzed using the Leica Application Suite (Leica Microsystems).

\section{Results}

Axon growth is inhibited by aggrecan

We cultured adult rat DRG neurons on the integrin substrate laminin, which is relevant because it is present in large amounts around Schwann cells and bands of Bungner, which support axon 
A
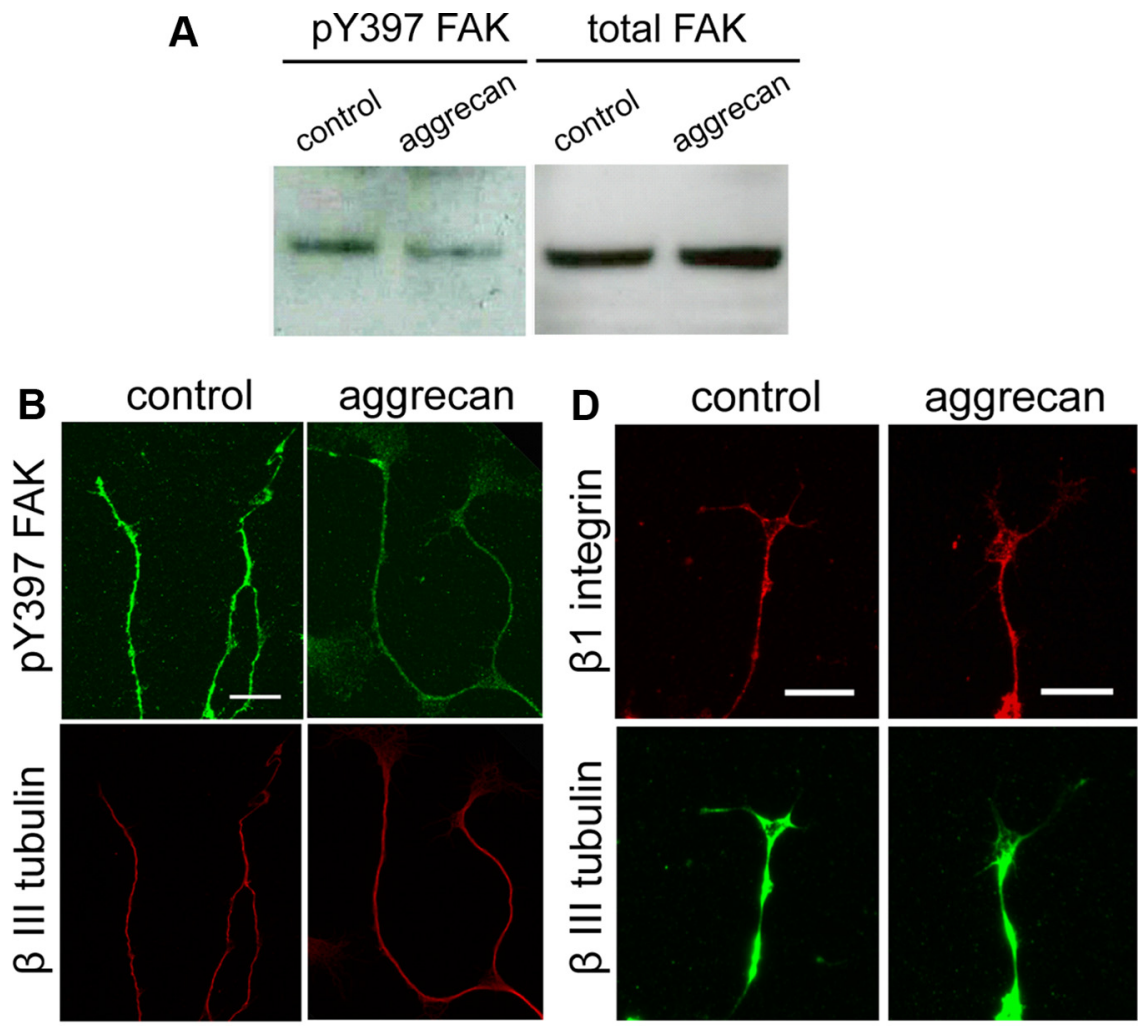

C

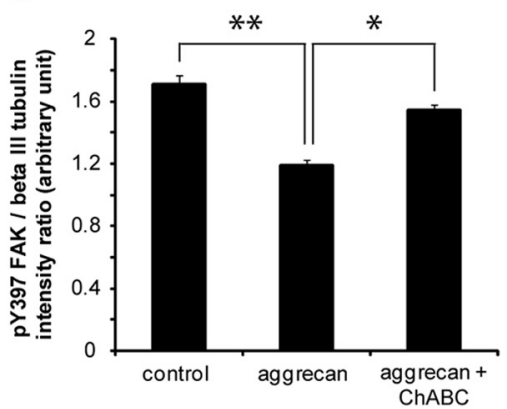

E

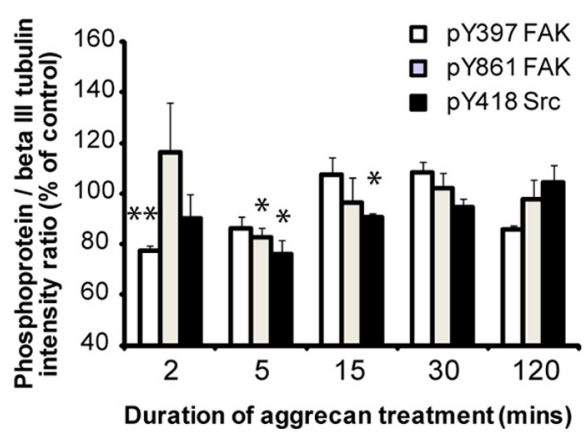

Figure 2. Effect of aggrecan on integrin signaling. $\boldsymbol{A}$, Aggrecan decreases pY397FAK level in DRG neurons, without altering total FAK level. $\boldsymbol{B}$, The same effect is observed in axons and growth cones, as analyzed by quantitative immunofluorescence. Scale bar, $20 \mu \mathrm{m}$. C, ChABC treatment of aggrecan eliminates its inhibitory effect on pY397 FAK. D, Aggrecan does not alter the level of $\beta 1$ integrin expressed on the surface of DRG axons. Scale bar, $15 \mu \mathrm{m}$. $\boldsymbol{E}$, The phosphorylation status of Y397 FAK, Y861 FAK, and Y418 Src residues undergoes sequential decreases after aggrecan treatment. pY397 FAK is maximally decreased at 2 min and pY861 at 5 min, while pY418 Src remains depressed for at least $15 \mathrm{~min}$. Data are mean $\pm \mathrm{SEM}$, analyzed with Student's $t$ test. ${ }^{* *} p<0.01$; ${ }^{*} p<0.05$.

regeneration after peripheral nerve injury. Axon growth after $20 \mathrm{~h}$ was assessed by measuring the percentage of neurons with axons, and the longest axon extended by each neuron. Axons grew profusely on laminin, the length of axons and the proportion of regenerating neurons varying with the concentration of laminin used for coating (Fig. $1 A$ ). Addition of aggrecan to the substrate inhibited axon growth at all laminin concentrations, although the effect gradually diminished with increasing laminin concentration (Fig. $1 B$ ). The addition of aggrecan to the substrate did not affect the level of laminin on the growth surfaces $(p=0.84, t$ test, $n=4)$ (Fig. 1C). That aggrecan inhibits growth even at saturating levels of laminin indicates that aggrecan is not acting simply by masking integrin binding sites on laminin. Removal of glycan chains from aggrecan by treatment with chondroitinase $\mathrm{ABC}$ (ChABC) $1 \mathrm{~h}$ before coating eliminated its axon growth-inhibitory effect $(p<0.01, t$ test, $n=3$ ) (Fig. 1D). This showed that the main inhibitory action of aggrecan is mediated through the glycan chains. The reduction in the glycan level in the substratum after ChABC treatment was clearly observed by immunostaining of CS-56 (Fig. 1E).

Aggrecan impairs integrin signaling We asked whether aggrecan-induced growth inhibition could be mediated via an effect on integrins. DRG neurons were cultured on laminin and allowed to extend axons for $20 \mathrm{~h}$. The neurons were then treated with either aggrecancontaining medium $(25 \mu \mathrm{g} / \mathrm{ml})$ or control medium for $2 \mathrm{~min}$. Western blotting was performed on proteins extracted from the neurons to determine the level of intracellular integrin signaling, on the basis of the level of phosphorylated-tyrosine-397 FAK (pY397 FAK) compared to total FAK. Aggrecan-treated neurons had a lower level of pY397 FAK, as compared to the control, with no difference in the level of total FAK (Fig. 2A). Since integrinrelated events that mediate axon outgrowth primarily take place near the ends of the axons, we performed a quantitative immunofluorescence assay to assess the changes of pY397 FAK in this region. As with the whole-cell Western blot, in the growth cone region, pY397 FAK level decreased by $32.3 \%(p<0.001$, $t$ test, $n=6)$ upon aggrecan treatment, without any changes in the total FAK level $(p=0.68, t$ test, $n=3$ ) (Fig. $2 B$ ). Treating aggrecan with $\mathrm{ChABC}$ before addition to the cultures significantly removed its inhibition on pY397 FAK level ( $p<0.05, t$ test, $n=$ 3) (Fig. 2C), suggesting that aggrecan impairs integrin signaling via its glycan chains.

To see whether the inhibition of integrin signaling was due to a change in the surface level of integrin, we examined the amount of integrin on the axonal surface after aggrecan treatment using quantitative immunohistochemistry on nonpermeabilized axons. Because DRG axon binding to laminin is mediated by $\beta 1$ integrins, the level of surface $\beta 1$ integrin was assessed. No difference in the surface $\beta 1$ integrin level was observed between aggrecan- and control medium-treated axons $(p=0.36, t$ test, $n=3$ ) (Fig. $2 D$ ), suggesting that aggrecan inhibits integrin signaling without altering surface integrin expression.

Investigating the changes in pY397 FAK over a longer time period, we observed that pY397 FAK level was maximally decreased at $2 \mathrm{~min}$, was less decreased at $5 \mathrm{~min}$, and returned to control level within $15 \mathrm{~min}(n=3)$ (Fig. $2 E$ ). Since the inhibitory effect of aggrecan on axon outgrowth is sustained and lasts much longer than 15 min, we asked whether integrin downstream signaling may exhibit a longer-lasting change. Downstream of 
tyrosine-397 residue phosphorylation, "outside-in" integrin signaling is propagated by the phosphorylation of other tyrosine residues on FAK, followed by the activation of other signaling molecules, such as Src. (Parsons, 2003; Mitra and Schlaepfer, 2006). We therefore examined the phosphorylation of tyrosine861 FAK (pY861 FAK) and tyrosine-418 Src (pY418 Src) after aggrecan treatment on DRG neurons. We found that the pY861 FAK level begins to decline $5 \mathrm{~min}$ after addition of aggrecan and returns to control level within $15 \min (n=3)$, while pY418 Src, which is further downstream, decreases after $5 \mathrm{~min}$, and stays at this depressed level for at least $15 \min (n=3)$ (Fig. 2E). These results are consistent with our hypothesis that aggrecan impairs integrin signaling. Moreover, they corroborate the sequential phosphorylation events known to occur during integrin signaling.

\section{Integrin activation increases axon} growth by enhancing integrin signaling To examine the effect of integrin activation on axon growth, we cultured DRG neurons on different laminin concentrations $(0.1-10 \mu \mathrm{g} / \mathrm{ml})$, in the presence or absence of manganese $(500 \mu \mathrm{M})$, and assessed the axon growth after $20 \mathrm{~h}$. Manganese is a potent integrin activator that holds integrin receptors in a conformation with a high ligand-binding affinity (Mould et al., 1995). At very low $(0.1 \mu \mathrm{g} /$ $\mathrm{ml})$ or high $(10 \mu \mathrm{g} / \mathrm{ml})$ laminin concentrations, addition of manganese did not alter the extent of axon growth. However, at low $(1 \mu \mathrm{g} / \mathrm{ml})$ laminin concentration, manganese treatment induced an increase in axon growth (percentage of neurons with axons, $22.0 \%$ to $30.2 \%, p<0.01$; axon length, $144.8 \mu \mathrm{m}$ to $184.5 \mu \mathrm{m}, p<$ $0.001, t$ test, $n=6$ ) (Fig. $3 A, B$ ). To verify that there is an integrin-activating effect of manganese, DRG cultures were immunostained live using a monoclonal antibody, 9EG7, which recognizes activated integrins (Bazzoni et al., 1995; Montanez et al., 2008). Neurons cultured in manganese-containing medium exhibited a higher level of 9EG7 immunostaining $(+54.2 \% ; p<0.01, t$ test, $n=3)$ on the axons compared to control (Fig. 3C), indicating more "active" integrins on the surface.

The growth-enhancing effect of manganese is probably mediated via increased "outside-in" integrin signaling. Therefore, the status of pY397 FAK in DRG neuron cultures after manganese treatment was assessed. From Western blot of whole-cell protein lysate, as well as quantitative immunofluorescence of axon/ growth cone regions, pY397 FAK level was markedly enhanced (quantitative immunofluorescence, $+56.1 \%$; $p<0.05, t$ test, $n=$ 3 ) in the presence of manganese (Fig. $3 D, E$ ), while total FAK level
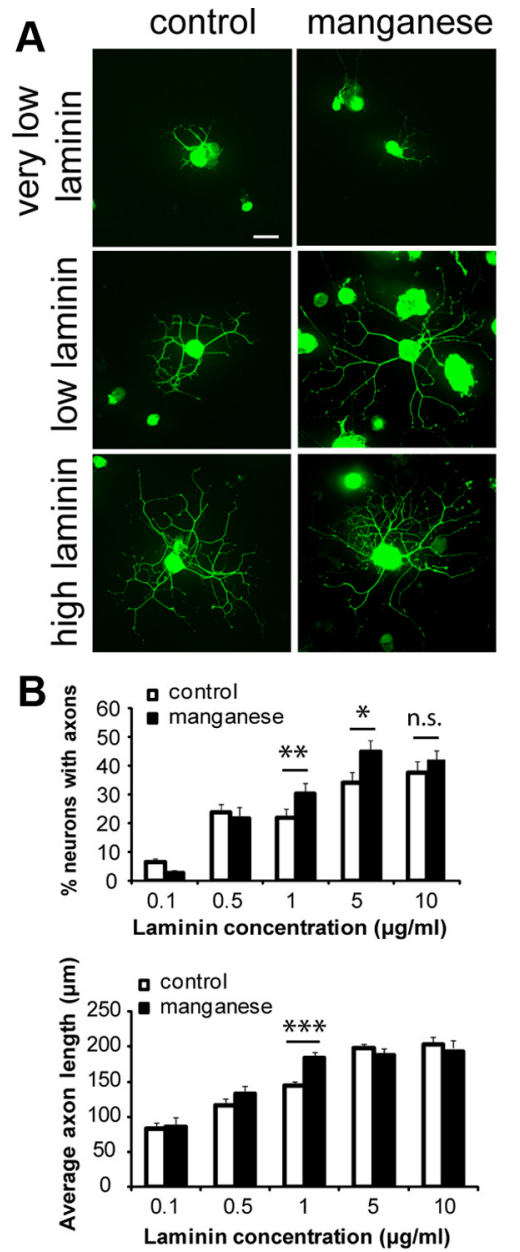

E control
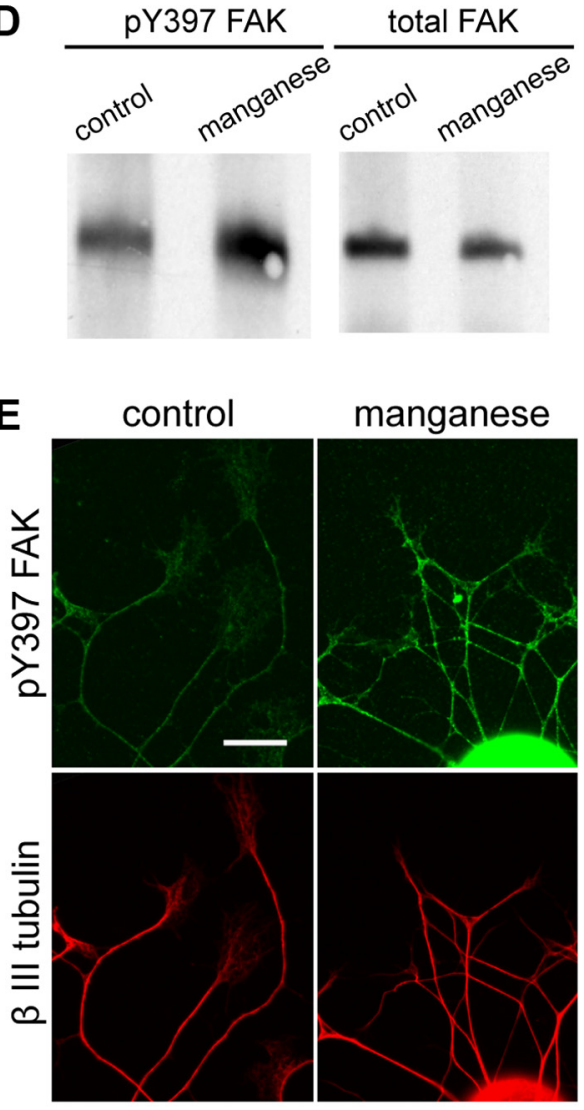

F

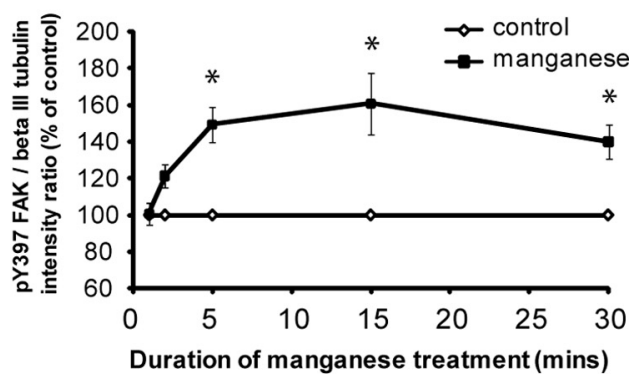

Figure 3. Effect of manganese-induced integrin activation on DRG neurons. $A, B$, Manganese-induced integrin activation enhances axon growth from cultured DRG neurons at low $(1 \mu \mathrm{g} / \mathrm{ml})$, but not very low $(0.1 \mu \mathrm{g} / \mathrm{ml})$ or high $(10 \mu \mathrm{g} / \mathrm{ml})$, conceninduces a rapid rise in $\mathrm{PY} 397 \mathrm{FAK}$ level, which reaches a statistically significant level within 5 min and plateaus thereafter. Data are mean \pm SEM, and analyzed with Student's $t$ test. ${ }^{* * *} p<0.001$; ${ }^{* *} p<0.01 ;{ }^{*} p<0.05$; n.s., not significant.

remained unchanged ( $p=0.80, t$ test, $n=3$ ). Furthermore, a time course study revealed that the pY397 FAK level began to increase after $2 \mathrm{~min}$ and reached a statistically significant level after $5 \min (p<0.05$, $t$ test, $n=3)$, before settling at a plateau of $\sim 150 \%$ of control level after 15 min (Fig. $3 F$ ).

\section{Integrin activation overcomes aggrecan-mediated growth} inhibition by enhancing integrin signaling

Since aggrecan inactivates integrins and decreases integrin signaling, we asked whether forced activation of integrins might overcome its inhibitory effect. DRG neurons were cultured for $20 \mathrm{~h}$ as before, then treated with aggrecan or mixed aggrecan-manganese solutions to assess the immediate effect on integrin signal- 
A

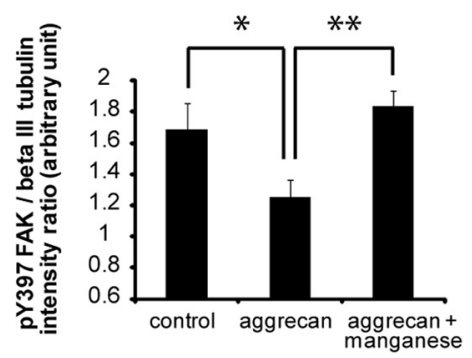

B
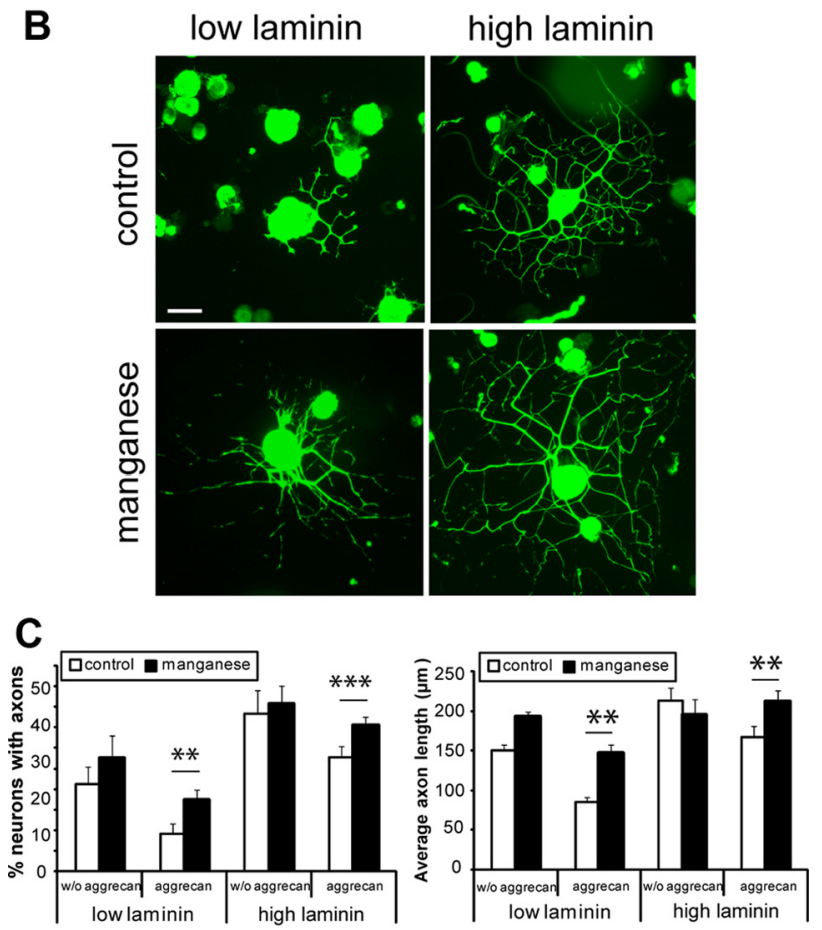

D laminin only
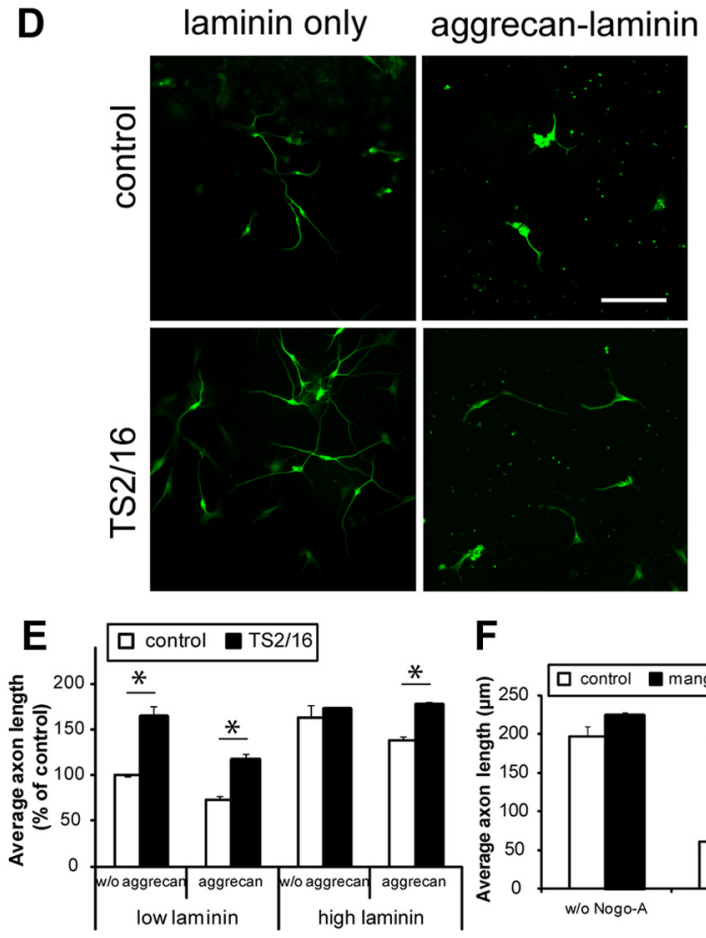

$\mathbf{F}$

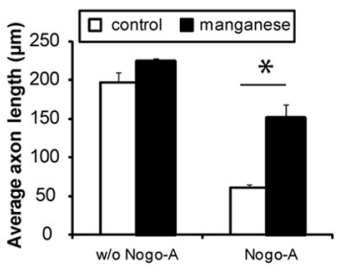

Figure 4. Effect of integrin activation on axon growth on inhibitory substrata. $\boldsymbol{A}$, Manganese treatment reverses the aggrecan-mediated inhibitory effect on integrin signaling. $\boldsymbol{B}-\boldsymbol{E}$, Addition of integrin activators, i.e., manganese to DRG neurons ( $\boldsymbol{B}, \boldsymbol{C}$, scale bar, $50 \mu \mathrm{m})$ and TS2/16 ing. Quantitative immunofluorescence analysis showed that the decrease in pY397 FAK level brought about by aggrecan was alleviated by manganese treatment $(p<0.01, t$ test, $n=5)$ (Fig. $4 A$ ).

The ability of integrin activation to rescue axon growth on inhibitory aggrecan was also assessed. To further dissect the potential differential effects at varying substrate concentrations, two laminin concentrations were selected to reflect "low" $(1 \mu \mathrm{g} / \mathrm{ml})$ or "high" $(10 \mu \mathrm{g} / \mathrm{ml})$ ligand availability. At both concentrations, aggrecan inhibited axon growth from DRG neurons, with the effect being stronger at the low laminin concentration. Addition of manganese restored the growth to control levels on both surfaces (percentage of neurons with axons: low laminin, 9.0\% to $17.3 \%, p<0.01$; high laminin, $27.7 \%$ to $35.5 \%, p<0.001$; axon length: low laminin, $85.6 \mu \mathrm{m}$ to $146.9 \mu \mathrm{m}, p<0.01$; high laminin, $167.4 \mu \mathrm{m}$ to $212.0 \mu \mathrm{m}, p<0.01, t$ test, $n=6$ ) (Fig. $4 B, C)$, suggesting that integrin activation enables neurons to overcome aggrecan-mediated inhibition to enhance axon growth.

The effects of manganese are not entirely specific to integrin activation. To confirm the growth-enhancing effect of manganese is attributable to integrin activation, we repeated our assays using a specific integrin activator, the monoclonal antibody TS2/16 (Tsuchida et al., 1997; Hu and Strittmatter, 2008). As the antibody specifically recognizes human integrins, we performed our experiments using motoneurons differentiated from hESCs, following an established protocol (Patani et al., 2009). The results we obtained were the same as those from DRG neurons. Activation of integrin, induced by TS2/16 antibody, alleviated the inhibitory effect of aggrecan on axon outgrowth, and enabled the motoneurons to extend longer axons on aggrecan-laminin substrates (low laminin, $72.5 \mu \mathrm{m}$ to $117.1 \mu \mathrm{m}, p<0.05$; high laminin, $138.1 \mu \mathrm{m}$ to $177.5 \mu \mathrm{m}, p<0.05, t$ test, $n=3$ ) (Fig. $4 D, E)$. This indicates that earlier results obtained with manganese treatment were attributable to its integrin-activating effect.

To confirm the generality of integrin modulation as a mechanism for inhibition of axon growth in the damaged CNS, we performed a similar experiment as previously reported ( $\mathrm{Hu}$ and Strittmatter, 2008), demonstrating that Nogo-A inhibits axon growth on laminin, but that this inhibition can be overcome by activation of integrins with manganese $(p<0.05, t$ test, $n=3)$ (Fig. $4 F$ ).

\section{Discussion}

The inhibitory effect of CSPGs on integrin signaling

There is evidence for various mechanisms mediating the inhibitory action of aggrecan and other CSPGs on axon growth. Several signaling pathways have been implicated, including Rho/ROCK activation (Monnier et al., 2003), PKC activation (Sivasankaran et al., 2004), and EGFR signaling (Koprivica et al., 2005). A recent paper proposed that the transmembrane protein tyrosine phosphatase $\sigma, \mathrm{PTP} \sigma$, is a receptor for CSPGs such as aggrecan and neurocan, and mediates the growth-inhibitory effect of these molecules (Shen et al., 2009). Our results suggest that these pathways may converge on the impairment of integrin function by affecting their activation state, resulting in decreased integrin signaling. An earlier study had showed that aggrecan inhibits NGF-supported axon assembly on laminin without affecting

to hESC-derived motoneurons ( $\boldsymbol{D}, \boldsymbol{E}$, scale bar, $100 \mu \mathrm{m}$ ) reverses the inhibition of aggrecan on axon growth. $\boldsymbol{F}$, Manganese reverses Nogo-A-mediated inhibition on axon growth. All data are mean \pm SEM, and analyzed with Student's $t$ test. ${ }^{* * *} p<0.001$; ${ }^{* *} p<0.01 ;{ }^{*} p<0.05$; n.S., not significant. 
NGF-induced ERK phosphorylation, which led the authors to infer that ECM-integrin signaling was a likely target for CSPGs (Zhou et al., 2006). Here, we demonstrate that aggrecan, via its glycan component, causes a suppression of integrin signaling, starting with a decrease in pY397 FAK level, maximal at 2 min, and decreasing thereafter. This is followed by decreases in further downstream phosphorylation events via pY861 FAK and pY418 Src. Crucially, levels of surface $\beta 1$ integrin were unaffected by aggrecan treatment. These changes are in agreement with previous data with regards to the sequence of phosphorylation events from integrin signaling (Parsons, 2003; Mitra and Schlaepfer, 2006). It is possible that interference with integrin signaling is a general mechanism for inhibition of axon growth. Interestingly, amino-Nogo also impairs integrin function and fibronectininduced pY397 FAK upregulation in cells ( $\mathrm{Hu}$ and Strittmatter, 2008). Semaphorins, which are also expressed in the damaged CNS, also impact integrin function (Pasterkamp and Giger, 2009).

\section{Integrin activation promotes axon growth by enhancing "outside-in" integrin signaling}

Earlier studies have demonstrated that integrin activation increases axon growth from PC-12 cells and mouse DRG neurons (Ivins et al., 2000; Lein et al., 2000). We found that this effect is dependent on substrate (laminin) concentration; i.e., integrin activation only enhances axon growth at low laminin concentrations but not on very low- or high-laminin substrata. A likely explanation is that when presented with very low concentrations of ligand, activation of integrins cannot override the paucity of ligands, while at very high ligand concentrations, most integrins are already activated and cannot be further activated. At low ligand concentrations, a high proportion of integrins are "inactive," allowing manganese to switch them into the "active" state (Lemons and Condic, 2006).

The increase in ligand-binding affinity conferred by integrin activation increases "outside-in" integrin signaling, as demonstrated by enhanced phosphorylation of Y397 of FAK. Integrin signaling enhancement in response to integrin activation was rapid with pY397 FAK starting to rise within 2 min of manganese addition, reaching a plateau in $5 \mathrm{~min}$ at $\sim 150 \%$ of the control level. That manganese induces a rapid integrin activation effect is in agreement with previous work using flow cytometry and quantitative immunofluorescence to detect activated integrins (Bazzoni et al., 1998; Zhao et al., 2005; Lemons and Condic, 2006), as well as functional assays (Nieswandt et al., 2007; Moser et al., 2008).

\section{Integrin activation allows axons to overcome inhibitory influences}

The inhibition of axon growth caused by CSPGs was successfully overcome by activating integrins in neuronal cultures, using both manganese and the integrin-activating antibody TS2/16 as the activating agents. This could result either from integrin activation overcoming a ligand-masking effect of CSPGs or through overcoming CSPG-mediated integrin inactivation. Our data speak against the first option: we grew axons in the presence of a large excess of laminin $(10 \mu \mathrm{g} / \mathrm{ml})$, yet aggrecan was still inhibitory at these concentrations and treatment with integrin activators (manganese or TS2/16) reversed this inhibition. We conclude that integrin activation enhances axon growth on a CSPGcontaining substratum by reversing the inactivation caused by the CSPG. Inhibition of axon growth through integrin inactiva- tion may be a general mechanism, because Nogo-A exerts part of its inhibitory effect in this way (Hu and Strittmatter, 2008).

Our results together with previous experiments demonstrate that molecules that inhibit axon regeneration in the CNS act in part by interfering with integrin function. Crucially, this inhibition can be overcome by enhancing the ligand-binding affinity of integrin receptors on axons through integrin activation. Activation of axonal integrins should therefore promote axon regeneration in the damaged CNS, where these inhibitors are upregulated (Silver and Miller, 2004; Xie and Zheng, 2008). We have shown that expression of an integrin that interacts with tenascin-C, a glycoprotein upregulated in the damaged CNS, can enhance axon regeneration after CNS damage (Andrews et al., 2009). Transgenic expression of this integrin, coupled with integrin activation, should be a powerful method of promoting axon regeneration in the damaged CNS.

\section{References}

Afshari FT, Kwok JC, White L, Fawcett JW (2010) Schwann cell migration is integrin-dependent and inhibited by astrocyte-produced aggrecan. Glia 58:857-869.

Andrews MR, Czvitkovich S, Dassie E, Vogelaar CF, Faissner A, Blits B, Gage FH, ffrench-Constant C, Fawcett JW (2009) Alpha9 integrin promotes neurite outgrowth on tenascin- $\mathrm{C}$ and enhances sensory axon regeneration. J Neurosci 29:5546-5557.

Asher RA, Scheibe RJ, Keiser HD, Bignami A (1995) On the existence of a cartilage-like proteoglycan and link proteins in the central nervous system. Glia 13:294-308.

Bazzoni G, Shih DT, Buck CA, Hemler ME (1995) Monoclonal antibody 9EG7 defines a novel beta 1 integrin epitope induced by soluble ligand and manganese, but inhibited by calcium. J Biol Chem 270:25570-25577.

Bazzoni G, Ma L, Blue ML, Hemler ME (1998) Divalent cations and ligands induce conformational changes that are highly divergent among beta 1 integrins. J Biol Chem 273:6670-6678.

Borisoff JF, Chan CC, Hiebert GW, Oschipok L, Robertson GS, Zamboni R, Steeves JD, Tetzlaff W (2003) Suppression of Rho-kinase activity promotes axonal growth on inhibitory CNS substrates. Mol Cell Neurosci 22:405-416.

Chan CC, Roberts CR, Steeves JD, Tetzlaff W (2008) Aggrecan components differentially modulate nerve growth factor-responsive and neurotrophin-3responsive dorsal root ganglion neurite growth. J Neurosci Res 86:581-592.

Harrington AE, Morris-Triggs SA, Ruotolo BT, Robinson CV, Ohnuma S, Hyvönen M (2006) Structural basis for the inhibition of activin signaling by follistatin. EMBO J 25:1035-1045.

Hu F, Strittmatter SM (2008) The N-terminal domain of Nogo-A inhibits cell adhesion and axonal outgrowth by an integrin-specific mechanism. J Neurosci 28:1262-1269.

Hynes RO (2002) Integrins: bidirectional, allosteric signaling machines. Cell 110:673-687.

Ivins JK, Yurchenco PD, Lander AD (2000) Regulation of neurite outgrowth by integrin activation. J Neurosci 20:6551-6560.

Koprivica V, Cho KS, Park JB, Yiu G, Atwal J, Gore B, Kim JA, Lin E, TessierLavigne M, Chen DF, He Z (2005) EGFR activation mediates inhibition of axon regeneration by myelin and chondroitin sulfate proteoglycans. Science 310:106-110.

Lein P, Gallagher PJ, Amodeo J, Howie H, Roth JA (2000) Manganese induces neurite outgrowth in PC12 cells via upregulation of alpha(v) integrins. Brain Res 885:220-230.

Lemons ML, Condic ML (2006) Combined integrin activation and intracellular cAMP cause Rho GTPase dependent growth cone collapse on laminin-1. Exp Neurol 202:324-335.

Lemons ML, Condic ML (2008) Integrin signaling is integral to regeneration. Exp Neurol 209:343-352.

Li H, Domowicz M, Hennig A, Schwartz NB (1996) S103L reactive chondroitin sulfate proteoglycan (aggrecan) mRNA expressed in developing chick brain and cartilage is encoded by a single gene. Brain Res Mol Brain Res 36:309-321.

Long KE, Lemmon V (2000) Dynamic regulation of cell adhesion molecules during axon outgrowth. J Neurobiol 44:230-245. 
Mitra SK, Schlaepfer DD (2006) Integrin-regulated FAK-Src signaling in normal and cancer cells. Curr Opin Cell Biol 18:516-523.

Monnier PP, Sierra A, Schwab JM, Henke-Fahle S, Mueller BK (2003) The Rho/ROCK pathway mediates neurite growth-inhibitory activity associated with the chondroitin sulfate proteoglycans of the CNS glial scar. Mol Cell Neurosci 22:319-330.

Montanez E, Ussar S, Schifferer M, Bösl M, Zent R, Moser M, Fässler R (2008) Kindlin-2 controls bidirectional signaling of integrins. Genes Dev 22:1325-1330.

Moser M, Nieswandt B, Ussar S, Pozgajova M, Fässler R (2008) Kindlin-3 is essential for integrin activation and platelet aggregation. Nat Med 14:325-330.

Mould AP, Akiyama SK, Humphries MJ (1995) Regulation of integrin alpha 5 beta 1-fibronectin interactions by divalent cations. Evidence for distinct classes of binding sites for $\mathrm{Mn}^{2+}, \mathrm{Mg}^{2+}$, and $\mathrm{Ca}^{2+}$. J Biol Chem 270:26270-26277.

Nieswandt B, Moser M, Pleines I, Varga-Szabo D, Monkley S, Critchley D, Fässler R (2007) Loss of talin1 in platelets abrogates integrin activation, platelet aggregation, and thrombus formation in vitro and in vivo. J Exp Med 204:3113-3118.

Parsons JT (2003) Focal adhesion kinase: the first ten years. J Cell Sci 116:1409-1416.

Pasterkamp RJ, Giger RJ (2009) Semaphorin function in neural plasticity and disease. Curr Opin Neurobiol 19:263-274.

Patani R, Compston A, Puddifoot CA, Wyllie DJ, Hardingham GE, Allen ND, Chandran S (2009) Activin/Nodal inhibition alone accelerates highly efficient neural conversion from human embryonic stem cells and imposes a caudal positional identity. PLoS One 4:e7327.

Previtali SC, Feltri ML, Archelos JJ, Quattrini A, Wrabetz L, Hartung H (2001) Role of integrins in the peripheral nervous system. Prog Neurobiol 64:35-49.
Reichardt LF, Bixby JL, Hall DE, Ignatius MJ, Neugebauer KM, Tomaselli KJ (1989) Integrins and cell adhesion molecules: neuronal receptors that regulate axon growth on extracellular matrices and cell surfaces. Dev Neurosci 11:332-347.

Shen Y, Tenney AP, Busch SA, Horn KP, Cuascut FX, Liu K, He Z, Silver J, Flanagan JG (2009) PTPsigma is a receptor for chondroitin sulfate proteoglycan, an inhibitor of neural regeneration. Science 326: 592-596.

Silver J, Miller JH (2004) Regeneration beyond the glial scar. Nat Rev Neurosci 5:146-156,

Sivasankaran R, Pei J, Wang KC, Zhang YP, Shields CB, Xu XM, He Z (2004) PKC mediates inhibitory effects of myelin and chondroitin sulfate proteoglycans on axonal regeneration. Nat Neurosci 7:261-268.

Song H, Poo M (2001) The cell biology of neuronal navigation. Nat Cell Biol 3:E81-E88.

Tang X, Davies JE, Davies SJ (2003) Changes in distribution, cell associations, and protein expression levels of NG2, neurocan, phosphacan, brevican, versican V2, and tenascin-C during acute to chronic maturation of spinal cord scar tissue. J Neurosci Res 71:427-444.

Tsuchida J, Ueki S, Saito Y, Takagi J (1997) Classification of 'activation' antibodies against integrin betal chain. FEBS Lett 416:212-216.

Xie F, Zheng B (2008) White matter inhibitors in CNS axon regeneration failure. Exp Neurol 209:302-312.

Zhao Z, Gruszczynska-Biegala J, Zolkiewska A (2005) ADP-ribosylation of integrin alpha7 modulates the binding of integrin alpha7beta1 to laminin. Biochem J 385:309-317.

Zhou FQ, Walzer M, Wu YH, Zhou J, Dedhar S, Snider WD (2006) Neurotrophins support regenerative axon assembly over CSPGs by an ECMintegrin-independent mechanism. J Cell Sci 119:2787-2796. 